\title{
The effect of GnRH analogues for pituitary suppression on ovarian response in repeated ovarian stimulation cycles
}

\author{
Mario Cavagna ${ }^{1}$, Daniela Paes de Almeida Ferreira Braga ${ }^{2,3}$, Fabio Biaggioni Lopes ${ }^{2}$, \\ Rita de Cássia Savio Figueira², Assumpto laconelli Jr², Edson Borges Jr2,3
}

${ }^{1}$ Women's Health Reference Center, Hospital Perola Byington, São Paulo, Brazil 2Fertility-Assisted Fertilization Center, São Paulo, Brazil

${ }^{3}$ Sapientiae Institute - Educational and Research Center in Assisted Reproduction, São Paulo, Brazil

Submitted: 4 March 2010

Accepted: 20 May 2010

Arch Med Sci 2011; 7, 3: 470-475

DOI: 10.5114/aoms.2011.23414

Copyright @ 2011 Termedia \& Banach

\begin{abstract}
Introduction: Ovarian stimulation is employed in assisted reproduction techniques in order to obtain as many oocytes as possible. The early rise in oestradiol levels may lead to the premature end of the respective cycle. In order to avoid such an effect, pituitary suppression has been employed. The aim of this study was to evaluate whether maintenance or replacement of the type of GnRH analogue (i.e., agonist or antagonist) employed for pituitary suppression in the consecutive intracytoplasmic sperm injection (ICSI) cycle would negatively influence oocyte quality and ICSI outcome.

Material and methods: A retrospective observational study was conducted including 181 women with primary infertility. Patients were divided into four different groups according to the GnRH analogue used for pituitary suppression in the first and consecutive cycle.

Results: When a GnRH agonist was employed for pituitary suppression in the first cycle, the consecutive cycle showed comparable outcomes when performed with either a GnRH agonist or a GnRH antagonist. When the first cycle was performed with a GnRH antagonist, the use of the GnRH agonist in the successive cycle led to an increased number of oocytes retrieved (7.5\% vs. $10.3 \%$, $p=0.032)$ and the production of a higher number of embryos ( $4.5 \% \mathrm{vs.} 6.3 \%$, $p=0.036)$.

Conclusions: When the first cycle is carried out with a GnRH antagonist, the use of a GnRH agonist in the successive cycle would lead to increased numbers of oocytes collected and embryos produced.
\end{abstract}

Key words: controlled ovarian stimulation, GnRH agonist, GnRH antagonist, intracytoplasmic sperm injection.

\section{Introduction}

Ovarian stimulation, a standard procedure used in assisted reproductive technologies [1], is employed aiming to stimulate the growth of several follicles and, consequently, obtain as many high quality oocytes as possible. In controlled ovarian stimulation (COS) protocols, the early rise in oestradiol levels may promote an extemporaneous LH surge, leading to the premature end of the respective cycle. In order to avoid such an effect, over the last

\author{
Corresponding author: \\ Edson Borges Jr MD, PhD \\ Av. Brigadeiro Luis Antonio \\ 4545. Zip: 01401-002 \\ São Paulo - SP, Brazil \\ Phone: 551130188181 \\ E-mail: edson@fertility.com.br
}


20 years, pituitary suppression has been employed. Initially, pituitary suppression was attempted using $\mathrm{GnRH}$ agonists and, more recently, GnRH antagonists were introduced [2, 3].

Previous studies indicated that $\mathrm{GnRH}$ antagonists were related to lower pregnancy and implantation rates [4-7]. However, recent studies do not support such conclusions [8-11], especially when avoiding initiation of ovarian stimulation in patients showing high progesterone levels $[12,13]$ and when providing LH activity supplementation during the late follicular phase [1].

Another issue that remains controversial is whether ovarian response is affected by consecutive COS. While some investigations have suggested that repeated COS cycles lead to a decrease in the number of collected oocytes [14], other studies do not agree with such an effect [15, 16]. The aim of the present investigation is to evaluate whether maintenance or replacement of the type of $\mathrm{GnRH}$ analogue (i.e., agonist or antagonist) employed for pituitary suppression in the consecutive intracytoplasmic sperm injection (ICSI) cycle would negatively influence the ICSI outcome.

\section{Material and methods}

\section{Experimental design}

We retrospectively evaluated 181 women with primary infertility undergoing two consecutive ICSI cycles within a 3-month interval. The patients included in the study had an unsuccessful result in the first attempt and no cryopreserved embryos for a thawed embryo transfer; therefore a second ICSI cycle was performed.

All patients included in the study underwent the second ICSI cycle after the first attempt had failed. Patients showing the following characteristics were excluded from the study: (i) Body mass index $\geq 30 \mathrm{~kg} / \mathrm{m}^{2}$, (ii) blood serum FSH $>10 \mathrm{mU} / \mathrm{ml}$ on day 3 of the menstrual cycle, (iii) presence of clinically significant systemic disease, (iv) presence of endometriosis grades III-IV, (v) previous ovarian surgery, and (vii) presence of polycystic ovarian syndrome.

The distribution of the infertility causes among the groups is described in Table I. Informed written consent in which patients agreed to share the outcomes of their own cycles for research purposes was obtained. The study was approved by the local institutional review board.

Patients were divided into four different groups according to the GnRH analogue used for pituitary suppression: (i) group I: $\mathrm{GnRH}$ agonist in the first and second cycles of $\operatorname{COS}(n=66)$; (ii) group II: $\mathrm{GnRH}$ agonist in the first cycle and $\mathrm{GnRH}$ antagonist in the second ( $n=52$ ); (iii) group III: GnRH antagonist in both the first and second cycles of $\cos (n=36)$; (iv) group IV: GnRH antagonist and $\mathrm{GnRH}$ agonist in the first and second cycle, respectively $(n=27)$.

The endpoints of this study were total dose of r-FSH administered, number of metaphase II (MII) oocytes retrieved, number of embryos produced, and ongoing pregnancy rates in the second attempt. An ongoing pregnancy is defined as an intrauterine pregnancy with the presence of fetal heart beats 11-12 weeks after embryo transfer.

\section{Controlled ovarian stimulation}

In cycles in which a GnRH agonist was employed, ovarian stimulation was performed as follows: $1 \mathrm{mg}$ leuprolide acetate (Lupron Kit ${ }^{\circledR}$, Abbott S.A. Societé Française des laboratories, Paris, France) was administered subcutaneously from day 18 of the previous menstrual cycle. After 12 to 14 days, the dose of GnRH agonist was reduced to $0.5 \mathrm{mg}$ and ovarian stimulation was achieved with a fixed daily dose of 225 IU of r-FSH (Gonal F®, Serono, Geneve, Switzerland).

In cycles in which a GnRH antagonist was used, ovarian stimulation started on days 2-3 of the menstrual cycle with a fixed dose of $225 \mathrm{IU}$ of $\mathrm{r}-\mathrm{FSH}$ on a daily basis. When at least one follicle $\geq 14 \mathrm{~mm}$ was visualized $0.25 \mathrm{mg}$ of cetrorelix acetate (Cetrotide $^{\circledR}$, Serono, Geneve, Switzerland) was administered subcutaneously, until the day of hCG triggering injection.

For both pituitary blockage regimens, when adequate follicular growth and serum oestradiol levels were observed, recombinant human chorionic gonadotrophin was administered to trigger the final follicular maturation. Oocytes were collected $35 \mathrm{~h}$ after hCG administration by transvaginal ultrasound ovum pick-up.

Table I. Distribution of infertility cause among the groups

\begin{tabular}{|lccccc|}
\hline Infertility cause & I $(n=66)$ & II $(n=52)$ & III $(n=36)$ & IV $(n=27)$ & Value of $p$ \\
\hline Male factor & $60.6 \%$ & $57.7 \%$ & $55.5 \%$ & $66.6 \%$ & 0.657 \\
\hline Idiopathic & $9.09 \%$ & $11.5 \%$ & $13.8 \%$ & $11.1 \%$ & 0.612 \\
\hline Poor response & $27.2 \%$ & $25.0 \%$ & $27.7 \%$ & $18.5 \%$ & 0.328 \\
\hline Other & $3.03 \%$ & $5.7 \%$ & $2.7 \%$ & $3.7 \%$ & 0.525 \\
\hline
\end{tabular}




\section{Sperm samples}

Ejaculated spermatozoa were obtained by masturbation after 3-5 days of ejaculatory abstinence. After liquefaction at room temperature, sperm samples were selected by swim up, which was performed by diluting semen samples in a Hepes-buffered medium (1:1; Irvine Scientific, Santa Ana, USA) and incubating at $37^{\circ} \mathrm{C}$ for $1 \mathrm{~h}$, allowing spermatozoa to move from the seminal plasma to the overlayered culture medium.

\section{Preparation of oocytes}

After retrieval, oocytes were placed in culture medium (G-1 $1^{\mathrm{TM}}-\mathrm{V} 1$, Vitrolife, Kungsbacka, Sweden), Vitrolife, Kungsbacka, Sweden) covered with mineral oil (Ovoil ${ }^{\mathrm{TM}}$, Vitrolife, Kungsbacka, Sweden) and incubated at $37^{\circ} \mathrm{C}$ in $6 \% \mathrm{CO}_{2}$ for $5 \mathrm{~h}$. Cumulus cells were removed with a $30 \mathrm{~s}$ exposure to a Hepesbuffered medium containing $80 \mathrm{IU} / \mathrm{ml}$ hyaluronidase (Irvine Scientific, Santa Ana, USA). Coronal cells were then manually removed using a finely drawn glass Pasteur pipette (Humagen Fertility Diagnostics, Charlottesville, Virginia, USA).

Denuded oocytes were then assessed for nuclear status. Oocytes showing the release of the first polar body were considered mature and used for ICSI.

\section{Intracytoplasmic sperm injection}

For ICSI, oocytes were placed individually in $4 \mu \mathrm{l}$ droplets of buffered medium (G-Mops ${ }^{\mathrm{TM}}$-V1, Vitrolife, Kungsbacka, Sweden). Sperm was placed in a central $4 \mu \mathrm{l}$ droplet of polyvinylpyrrolidone solution (PVP, Irvine Scientific, Santa Ana, USA) in a $60 \times$ $15 \mathrm{Mm}$ plastic culture dish (Corning, Massachusetts, USA) covered with warm mineral oil (Ovoil ${ }^{\mathrm{TM}}$, Vitrolife, Kungsbacka, Sweden). Sperm injection was carried out on the heated stage $\left(37^{\circ} \mathrm{C}\right)$ of an inverted microscope (Eclipse TE 300; Nikon ${ }^{\circledR}$, Tokyo, Japan) $40 \mathrm{~h}$ after hCG trigger

\section{Statistical analysis}

Categorical variables were analysed using $\chi^{2}$ or Fisher test as appropriate. Student's $t$-test was employed when the numerical variables showed a normal distribution. Results were then described as mean and standard deviation. Non-parametric analysis (Mann-Whitney test) was performed for non-normally distributed data. In this case, median and range were used for results description.

Results were considered to be significant at the $5 \%$ critical level $(p<0.05)$. Data analysis was carried out using Minitab (version 14).

\section{Results}

In both groups submitted to the $\mathrm{GnRH}$ agonist in the first cycle, patients' mean ages were 34.29 \pm 4.69 and $35.85 \pm 3.81$ years for groups I and II, respectively ( $p=0.658$ ). Also for these groups, the interval between cycles was $68.23 \pm 15.68$ and 56.40 \pm 19.25 days for groups I and II, respectively $(p=0.494)$. The outcomes of ovarian stimulation in these groups are presented in Table II.

For patients in group I, no differences were found between the first and second cycle for any variable. When patients were treated with the GnRH agonist in the first cycle and subsequently with the antagonist in the second cycle (group II), a significant increase in the number of retrieved MII oocytes was observed. This, however, was not followed by an increase in the number of embryos produced. No differences were found in pregnancy rates between groups I and II (21.2\% vs. $28.8 \%$, respectively; $p=0.398$; Table III).

The mean ages of patients submitted to the $\mathrm{GnRH}$ antagonist in the first cycle were $36.9 \pm 5.0$ and $35.9 \pm 5.0$ years for groups III and IV, respectively $(p=0.459)$. Moreover, the mean intervals between cycles were $54.7 \pm 16.4$ and $69.7 \pm 23.5$ days for groups III and IV, respectively ( $p=0.254)$.

The outcomes of ovarian stimulation in these groups are presented in Table II. A significant increase in r-FSH dose administered was observed in group III when comparing the first and the second cycles. When, instead of the GnRH antagonist of the first cycle, an agonist was used in the second cycle (group IV), significant increases in the number of retrieved MII oocytes and in the number of embryos produced were observed. However, no differences were found between groups III and IV in pregnancy rates (19.5\% vs. $22.2 \%$, respectively; $p=0.877$; Table III).

Regarding the number of transferred embryos, no significant difference was found when comparing the first and second attempt for groups I, II, III and IV (Table II).

\section{Discussion}

The response to ovarian stimulation depends on several parameters such as woman's age and ovarian reserve status. Whether or not repeated and consecutive COS cycles impair the outcomes of the procedure is controversial. Kolibianakis et al. [14] conducted a retrospective analysis with 3,249 patients submitted to at least two COS cycles, and registered an age-independent deterioration of ovarian response across repeated cycles. On the other hand, de Boer et al. [15], studying 330 women undergoing consecutive ICSI cycles, observed no decrease in the number of retrieved oocytes over subsequent cycles. Moreover, Caligara et al. [17] reported that, in young oocyte donor women, successive COS cycles do not impair ovarian response in terms of the number of oocytes obtained. 
Table II. Outcomes of ovarian stimulation in patients submitted to two consecutive ICSI cycles, based on the nature of the GnRH analogue (agonist and antagonist) used for pituitary suppression in each cycle

\begin{tabular}{|c|c|c|c|c|c|c|c|}
\hline & \multirow[t]{2}{*}{$n$} & \multirow[t]{2}{*}{ Groups } & \multicolumn{2}{|c|}{ First cycle } & \multicolumn{2}{|c|}{ Second cycle } & \multirow[t]{2}{*}{ Value of $p$} \\
\hline & & & Agonist & Antagonist & Agonist & Antagonist & \\
\hline \multirow{4}{*}{$\begin{array}{l}\text { Total r-FSH } \\
\text { dose } \\
\text { administered (IU) }\end{array}$} & 66 & I & $2,447.7 \pm 628.9$ & - & $2,583.7 \pm 765.6$ & - & 0.121 \\
\hline & 52 & II & $2,628.8 \pm 542.9$ & - & - & $2,790.4 \pm 626.9$ & 0.078 \\
\hline & 36 & III & - & $2,509.8 \pm 593.2$ & - & $2,888.2 \pm 761.8$ & 0.001 \\
\hline & 27 & IV & - & $2,652.0 \pm 666.9$ & $2,831.0 \pm 796.0$ & - & 0.179 \\
\hline \multirow{4}{*}{$\begin{array}{l}\text { Number of MII } \\
\text { oocytes collected }\end{array}$} & 66 & 1 & $8.9 \pm 6.8$ & - & $9.5 \pm 6.7$ & - & 0.288 \\
\hline & 52 & II & $5.0 \pm 3.7$ & - & - & $6.5 \pm 4.7$ & 0.024 \\
\hline & 36 & III & - & $7.2 \pm 5.5$ & - & $7.3 \pm 6.4$ & 0.909 \\
\hline & 27 & IV & - & $7.5 \pm 6.9$ & $10.3 \pm 8.4$ & - & 0.032 \\
\hline \multirow{4}{*}{$\begin{array}{l}\text { Number of } \\
\text { embryos } \\
\text { produced }\end{array}$} & 66 & 1 & $4.8 \pm 3.2$ & - & $5.8 \pm 4.2$ & - & 0.088 \\
\hline & 52 & II & $3.0 \pm 2.3$ & - & - & $3.8 \pm 2.3$ & 0.090 \\
\hline & 36 & III & - & $4.4 \pm 3.0$ & - & $4.8 \pm 3.7$ & 0.440 \\
\hline & 27 & IV & - & $4.5 \pm 3.5$ & $6.3 \pm 5.2$ & - & 0.036 \\
\hline \multirow{4}{*}{$\begin{array}{l}\text { Number of } \\
\text { transferred } \\
\text { embryos }\end{array}$} & 66 & 1 & $3.07 \pm 0.15$ & & $3.05 \pm 0.15$ & & 0.248 \\
\hline & 52 & II & $2.46 \pm 0.33$ & & & $2.76 \pm 0.41$ & 0.367 \\
\hline & 36 & III & & $2.93 \pm 0.26$ & & $3.01 \pm 0.23$ & 0.389 \\
\hline & 27 & IV & & $2.80 \pm 0.28$ & $2.80 \pm 0.26$ & & 0.452 \\
\hline
\end{tabular}

The present study aimed to investigate whether consecutive COS impairs the outcome of ICSI cycles, requiring higher amounts of $\mathrm{r}$ - $\mathrm{FSH}$ or promoting a lower ovarian response, in terms of MII oocytes retrieved and number of embryos produced. Our investigation studied not only the effects of repeating the cycle within 3 months, but also whether by modifying the nature of GnRH analogue used to achieve pituitary suppression, different outcomes could be achieved.

According to our results, patients submitted to the $\mathrm{GnRH}$ agonist in the first cycle suffered no deleterious effects regarding ovarian stimulation outcomes when a second ICSI cycle was performed within 3 months. In addition, we observed that when a GnRH agonist is used in the first attempt, the use of either a GnRH agonist or an antagonist in the second cycle leads to similar outcomes. However, when patients were submitted to the $\mathrm{GnRH}$ agonist in the first cycle followed by an antagonist in the second (group II), the number of MII oocytes retrieved was higher when compared to the GnRH agonist in both cycles (group I).

In groups in which pituitary suppression in the first cycle was performed with the GnRH antagonist, an increase in $\mathrm{r}$-FSH requirements was observed when the antagonist was maintained in the second attempt, despite previous experiments indicating that the use of GnRH antagonist in ovarian stimulation protocols is associated with lower requirements of FSH (Tarlatzis and Kolibianakis, 2007; Kurzawa et al., 2008). Even with increased
Table III. Pregnancy rates in patients submitted to two consecutive ICSI cycles, based on the nature of the GnRH analogue (agonist and antagonist) used for pituitary suppression in each cycle

\begin{tabular}{|lccc|}
\hline Groups & First cycle & Second cycle & Pregnancy rate \\
\hline I & Agonist & Agonist & $14 / 66(21.21 \%)$ \\
\hline II & Agonist & Antagonist & $15 / 52(28.84 \%)$ \\
\hline III & Antagonist & Antagonist & $7 / 36(19.5 \%)$ \\
\hline IV & Antagonist & Agonist & $6 / 27(22.2 \%)$ \\
\hline
\end{tabular}

amounts of $\mathrm{r}$-FSH in the second cycle with the $\mathrm{GnRH}$ antagonist, no differences were found in the number of oocytes retrieved and embryo production. On the other hand, when the GnRH antagonist used in the first cycle is replaced by an agonist in the consecutive cycle, a higher number of MII oocytes collected and a higher number of embryos produced were observed.

In fact, in two situations, an increased number of retrieved MII oocytes could be observed. The first was when the pituitary blockage was performed initially through a GnRH agonist and subsequently using a GnRH antagonist. In this case, however, the number of embryos was not higher. In the second situation the use of a GnRH antagonist followed by a GnRH agonist led to a higher number of MII and also a higher number of embryos.

$\mathrm{GnRH}$ agonists have the same effect on gonadotropin release after binding the receptor as native $\mathrm{GnRH}$. The main difference of $\mathrm{GnRH}$ agonists 
used in clinical practice, in comparison with native $\mathrm{GnRH}$, is that the half-life time and the bioavailability are prolonged, due to increased lipophilicity [18]. The continuous administration of $\mathrm{GnRH}$ agonists (daily or depot application) initially causes LH and FSH hypersecretion (flare-up), which is followed after a period of about 10 days by desensitization of the pituitary and profound suppression of LH and FSH [19]. The lack of pulsatile secretion of $\mathrm{LH}$ and $\mathrm{FSH}$ during long-term treatment with $\mathrm{GnRH}$ agonists results in the arrest of follicular growth at the gonadotrophindependent stage [20, 21].

Unlike GnRH agonists, the antagonists do not induce an initial hypersecretion of gonadotropins but instead cause an immediate and rapid reversible suppression of gonadotropin secretion. The principal mechanism of action of GnRH antagonists is competitive occupancy of the GnRH receptor. Their administration results in the suppression of $\mathrm{LH}$ (about 70\%) and FSH (about 30\%) serum levels after about $6 \mathrm{~h}$ [22-24].

Our results demonstrated that different protocols for pituitary blockage, in two consecutive cycles, have positive effects on the response to the COS. Why repetitive pituitary blockage protocols may have detrimental effects on the gonadotrophs is still a matter of debate. However, we suggest that the different mechanism of action of the two $\mathrm{GnRH}$ analogues may be responsible for the improved response to the COS.

Regarding the requirements of $\mathrm{r}-\mathrm{FSH}$, we also observed an increase in the total dose administered, although statistical significance was not achieved. This could be due to a higher inhibitory effect of the GnRH antagonist in ovaries on the consecutive cycle, since it is well established that specific $\mathrm{GnRH}$ receptors are present in human granulosa cells [25].

Both forms of $\mathrm{GnRH}$ receptors, GnRH I and $\mathrm{GnRH}$ II, are expressed in various compartments of the human ovary, so that $\mathrm{GnRH}$ and its analogues not only influence steroidogenesis, but also induce a decrease in proliferation and promote apoptosis of the ovarian cells [26]. When pituitary suppression is performed with a GnRH antagonist, the inhibitory effects of the analogue on the granulosa cells are more pronounced, and a second consecutive cycle would require higher amounts of $\mathrm{r}$-FSH to stimulate follicular development. This hypothesis is corroborated by the findings of Giampietro et al. [27], who compared the levels of apoptosis in granulosa cells from women treated with a GnRH agonist or a GnRH antagonist and found that the mean concentrations of oestradiol, testosterone and progesterone in the follicular fluid were significantly lower in the GnRH antagonist treated group.
Even if we cannot exclude a possible bias associated with the retrospective analysis of the data, our findings suggest that, when a COS cycle with GnRH antagonist has an unsuccessful pregnancy outcome, a consecutive cycle performed within 3 months should be repeated switching to the GnRH agonist protocol, in order to achieve better outcomes, at least with regard to the number of oocytes collected and embryos produced.

In conclusion, the data of the present investigation suggest that there are no deleterious effects of consecutive ovarian stimulation cycles, in terms of retrieved oocytes and embryo production. When a $\mathrm{GnRH}$ agonist is employed for pituitary suppression in the first cycle, the consecutive cycle shows comparable outcomes when performed with either a GnRH agonist or a GnRH antagonist. On the other hand, when the first cycle is carried out with a GnRH antagonist, our data suggest that the use of a GnRH agonist in the successive cycle may promote the retrieval of a higher number of oocytes and the consequent production of a higher number of embryos.

\section{References}

1. Baruffi RL, Mauri AL, Petersen CG, et al. Recombinant LH supplementation to recombinant FSH during induced ovarian stimulation in the $\mathrm{GnRH}$-antagonist protocol: a meta-analysis. Reprod Biomed Online 2007; 14: 14-25.

2. Albano C, Smitz J, Camus M, et al. Comparison of different doses of gonadotropin-releasing hormone antagonist Cetrorelix during controlled ovarian hyperstimulation. Fertil Steril 1997; 67: 917-22.

3. Porter RN, Smith W, Craft IL, Abdulwahid NA, Jacobs HS. Induction of ovulation for in-vitro fertilisation using buserelin and gonadotropins. Lancet 1984; 2: 1284-5.

4. Hernandez ER. Embryo implantation and $\mathrm{GnRH}$ antagonists: embryo implantation: the Rubicon for $\mathrm{GnRH}$ antagonists. Hum Reprod 2000; 15: 1211-6.

5. Ludwig $M$, Katalinic A, Diedrich K. Use of $\mathrm{GnRH}$ antagonists in ovarian stimulation for assisted reproductive technologies compared to the long protocol. Meta-analysis. Arch Gynecol Obstet 2001; 265: 175-82.

6. Al-Inany HG, Abou-Setta AM, Aboulghar M. Gonadotrophin-releasing hormone antagonists for assisted conception: a Cochrane review. Reprod Biomed Online 2007; 14: 640-9.

7. Lindheim SR, Morales AJ. GnRH antagonists followed by a decline in serum estradiol results in adverse outcomes in donor oocyte cycles. Hum Reprod 2003; 18: 2048-51.

8. Bodri D, Vernaeve V, Guillen JJ, et al. Comparison between a GnRH antagonist and a GnRH agonist flare-up protocol in oocyte donors: a randomized clinical trial. Hum Reprod 2006; 21: 2246-51.

9. Kolibianakis EM, Collins J, Tarlatzis BC, et al. Among patients treated for IVF with gonadotrophins and GnRH analogues, is the probability of live birth dependent on the type of analogue used? A systematic review and meta-analysis. Hum Reprod Update 2006; 12: 651-71.

10. Moraloglu O, Kilic S, Karayalcin R, et al. Comparison of GnRH agonists and antagonists in normoresponder IVF/ICSI in Turkish female patients. Adv Ther 2008; 25: 266-73. 
11. Wei AY, Mijal KA, Christianson MS, Schouweiler CM, Lindheim SR. Comparison of GnRH antagonists and flareup GnRH agonists in donor oocyte cycles. J Reprod Med 2008; 53: 147-50.

12. Kolibianakis EM, Zikopoulos K, Smitz J, et al. Elevated progesterone at initiation of stimulation is associated with a lower ongoing pregnancy rate after IVF using GnRH antagonists. Hum Reprod 2004; 19: 1525-9.

13. Kolibianakis EM, Bourgain C, Papanikolaou EG, et al. Prolongation of follicular phase by delaying hCG administration results in a higher incidence of endometrial advancement on the day of oocyte retrieval in $\mathrm{GnRH}$ antagonist cycles. Hum Reprod 2005; 20: 2453-6.

14. Kolibianakis E, Osmanagaoglu K, Camus M, et al. Effect of repeated assisted reproductive technology cycles on ovarian response. Fertil Steril 2002; 77: 967-70.

15. de Boer EJ, Den Tonkelaar I, Burger CW, et al. The number of retrieved oocytes does not decrease during consecutive gonadotrophin-stimulated IVF cycles. Hum Reprod 2004; 19: 899-904.

16. Hoveyda F, Engmann L, Steele J, Lopez Bernal A, Barlow $\mathrm{DH}$. Ovarian response in three consecutive in vitro fertilization cycles. Fertil Steril 2002; 77: 706-10.

17. Caligara C, Navarro J, Vargas G, et al. The effect of repeated controlled ovarian stimulation in donors. Hum Reprod 2001; 16: 2320-3.

18. Karten MJ, Rivier JE. Gonadotropin-releasing hormone analog design. Structure-function studies toward the development of agonists and antagonists: rationale and perspective. Endocr Rev 1986; 7: 44-66.

19. Huirne JA, Lambalk, CB. Gonadotropin-releasing-hormonereceptor antagonists. Lancet 2001; 358: 1793-803.

20. Gong JG, Campbell BK, Bramley TA, et al. Suppression in the secretion of follicle-stimulating hormone and luteinizing hormone, and ovarian follicle development in heifers continuously infused with a gonadotropinreleasing hormone agonist. Biol Reprod 1996; 55: 68-74.

21. D'Occhio MJ, Fordyce G, Whyte TR, Aspden WJ, Trigg TE. Reproductive responses of cattle to $\mathrm{GnRH}$ agonists. Anim Reprod Sci 2000; 60-61: 433-42.

22. Schally AV. Luteinizing hormone-releasing hormone analogs: their impact on the control of tumorigenesis. Peptides 1999; 20: 1247-62.

23. Rivier J, Porter J, Hoeger C, et al. Gonadotropin-releasing hormone antagonists with $\mathrm{N}$ omega-triazolylornithine, -lysine, or - $\mathrm{p}$-aminophenylalanine residues at positions 5 and 6. J Med Chem 1992; 35: 4270-8.

24. NestorJr JJ, Tahilramani R, Ho TL, et al. Potent gonadotropin releasing hormone antagonists with low histamine-releasing activity. J Med Chem 1992; 35: 3942-8.

25. Ortmann O, Weiss JM, Diedrich K. Embryo implantation and GnRH antagonists: ovarian actions of $\mathrm{GnRH}$ antagonists. Hum Reprod 2001; 16: 608-11.

26. Metallinou C, Asimakopoulos B, Schroer A, Nikolettos N. Gonadotropin-releasing hormone in the ovary. Reprod Sci 2007; 14: 737-49.

27. Giampietro F, Sancilio S, Tiboni GM, Rana RA, Di Pietro R. Levels of apoptosis in human granulosa cells seem to be comparable after therapy with a gonadotropin-releasing hormone agonist or antagonist. Fertil Steril 2006; 85: 412-9. 